\section{RMD Open}

Rheumatic \& Musculoskeletal Diseases

\title{
Long-term efficacy and predictors of remission following adalimumab treatment in peripheral spondyloarthritis: 3-year results from ABILITY-2
}

To cite: Van den Bosch F, Mease PJ, Sieper J, et al. Longterm efficacy and predictors of remission following adalimumab treatment in peripheral spondyloarthritis: 3-year results from ABILITY-2. RMD Open 2018;4:e000566. doi:10.1136/ rmdopen-2017-000566

- Prepublication history for this paper is available online. To view these files, please visit the journal online (http://dx.doi. org/10.1136/rmdopen-2017000566).

Received 25 August 2017 Revised 21 December 2017 Accepted 9 January 2018

\section{ABSTRACT}

Objectives Describe efficacy and safety of 3 years of adalimumab treatment in patients with peripheral spondyloarthritis ( $\mathrm{pSpA}$ ) and identify predictors of remission.

Methods Patients with $\mathrm{pSpA}$ were randomised to adalimumab $40 \mathrm{mg}$ every other week or placebo for 12 weeks; a 144-week open-label extension followed (NCT01064856). Remission was assessed by the Peripheral SpA Response Criteria (PSpARC) and Ankylosing Spondylitis Disease Activity Score inactive disease (ASDAS ID). Logistic regression analyses were performed to determine predictors of remission at 1 and 3 years and sustained remission ( $\geq 24$ consecutive weeks).

Results In 165 patients, ASDAS ID was achieved by $47 \%$ at 1 year and $39 \%$ at 3 years; $36 \%$ and $33 \%$ achieved PSpARC remission, respectively. Sustained ASDAS ID and PSpARC remission were achieved by $52 \%$ (86/165) and $42 \%$ (70/165) of patients, respectively. Achieving ASDAS ID at week 12 significantly predicted 1 year (OR, $8.64(95 \% \mathrm{Cl}$ 2.97 to 25.14$)$ ), 3 year (OR, $36.12(95 \% \mathrm{Cl} 2.29$ to 569.08$)$ ) and sustained ASDAS ID (OR, 8.01 (95\% Cl 2.47 to 25.97$)$ ); achieving PSpARC remission at week 12 consistently predicted 1 year (OR, 6.47 (95\% $\mathrm{Cl} 1.91$ to 21.95$))$, 3 years (OR, 15.66 (95\% Cl 4.19 to 58.56)) and sustained PSpARC remission (OR, $20.27(95 \% \mathrm{Cl} 5.37$ to 76.46$))$. No baseline variables consistently predicted 1-year or 3-year remission or sustained remission. The safety profile of adalimumab was consistent with observations in other SpA disease indications.

Conclusions In patients with $\mathrm{pSpA}$, early response to adalimumab, but not baseline demographics or disease characteristics, was a better predictor of long-term remission.

\section{INTRODUCTION}

For numbered affiliations see end of article.

\section{Correspondence to}

Professor Philip J Mease; pmease@philipmease.com
Peripheral spondyloarthritis (pSpA) typically presents with peripheral arthritis that is usually asymmetric and predominantly involves the lower limbs, enthesitis and/ or dactylitis; this can be accompanied by

\section{Key messages}

What is already known about this subject?

- To date, only a few other studies have evaluated the efficacy of tumour necrosis factor (TNF) inhibitors in patients with active peripheral spondyloarthritis (pSpA), and no long-term safety and efficacy data are available for $\mathrm{pSpA}$.

What does this study add?

- In the ABILITY-2 study in patients with pSpA, the safety and efficacy of adalimumab were maintained through 3 years.

- Up to half of patients experienced sustained remission and remission at 1 and 3 years.

- Achievement of remission at week 12 was the strongest predictor of sustained and long-term remission.

How might this impact on clinical practice?

- Identification of factors that predict long-term and sustained remission in patients with $\mathrm{pSpA}$ after treatment with TNF inhibitors may facilitate clinical decisions regarding patient therapy.

additional extra-articular disease manifestations, such as uveitis, psoriasis and Crohn's disease. ${ }^{1}$ In contrast to other rheumatic diseases, such as rheumatoid arthritis (RA), psoriatic arthritis (PsA) and ankylosing spondylitis (AS) / axial SpA, long-term efficacy data in $\mathrm{pSpA}$ are very limited.

Recently, the ABILITY-2 study demonstrated the superior efficacy of adalimumab versus placebo over 12 weeks in patients with pSpA. ${ }^{2}$ To date, only a few other studies have evaluated the efficacy of tumour necrosis factor (TNF) inhibitors in patients with active $\mathrm{pSpA}^{3}{ }^{3}$ In a small $(\mathrm{n}=40)$ investigator-initiated placebo-controlled trial, patients 
Table 1 Baseline demographics and disease characteristics

\begin{tabular}{lc}
\hline Characteristic, mean \pm SD* $^{*}$ & $\begin{array}{c}\text { Adalimumab } \\
\mathbf{N}=165\end{array}$ \\
\hline Age, years & $40.6 \pm 11.9$ \\
\hline Women, $\mathrm{n}(\%)$ & $90(55)$ \\
\hline BMI (kg/m²) & $26.6 \pm 5.8$ \\
\hline Smoker, yes, $\mathrm{n}(\%)$ & $39(24)$ \\
\hline Symptom duration, years & $7.24 \pm 7.1$ \\
\hline HLA-B27-positive, $\mathrm{n}(\%) \dagger$ & $103(63)$ \\
\hline Prior DMARD use, $\mathrm{n}(\%)$ & $115(70)$ \\
\hline Concomitant DMARD use, $\mathrm{n}$ (\%) & $79(48)$ \\
\hline Elevated hs-CRP, $\mathrm{n}(\%)$ & $72(44)$ \\
\hline Swollen joint count (0-76) & $6.7 \pm 6.9$ \\
\hline Tender joint count (0-78) & $13.3 \pm 14.5$ \\
\hline Physician Global Assessment of disease & $58.7 \pm 15.3$ \\
activity (0-100) & \\
\hline Patient Global Assessment of pain (0-100) & $64.9 \pm 14.9$ \\
\hline Patient Global Assessment of disease & $65.8 \pm 15.5$ \\
activity (0-100) & \\
\hline Enthesitis count (0-29) & $7.0 \pm 6.8$ \\
\hline Dactylitis count (0-20) & $0.5 \pm 1.1$ \\
\hline BASDAl (0-10) & $5.62 \pm 1.7$ \\
\hline ASDAS & $2.99 \pm 0.8$ \\
\hline & \\
\hline
\end{tabular}

*Unless otherwise noted.

$\dagger \mathrm{n}=164$.

ASDAS, Ankylosing Spondylitis Disease Activity Score; BASDAI, Bath Ankylosing Spondylitis Disease Activity Index; BMI, body mass index; DMARD, disease-modifying antirheumatic drug; HLA, human leucocyte antigen; hs-CRP, high-sensitivity C-reactive protein.

received 12 weeks of adalimumab treatment followed by a 12-week open-label extension and a 16-week follow-up without active treatment. ${ }^{45}$ Another 12-week, doubleblind, placebo-controlled trial evaluated infliximab in patients with active SpA, including patients with $\mathrm{pSpA}{ }^{3}$ These two studies classified patients using the European Spondyloarthropathy Study Group criteria, ${ }^{6}$ whereas ABILITY-2 used Assessment of SpondyloArthritis international Society (ASAS) classification criteria. ${ }^{1}$

Currently, no long-term safety and efficacy data are available for pSpA. Furthermore, studies in other rheumatic diseases, including $\mathrm{RA},{ }^{7-13} \mathrm{PsA}^{1415}$ and $\mathrm{AS},{ }^{16}$ have evaluated predictors of short-term and long-term remission, but such data are lacking in pSpA. Here we report the long-term clinical efficacy and safety data from the ABILITY-2 study through the end of 3 years with a special focus on evaluation of potential predictors of long-term remission.

\section{METHODS}

The ABILITY-2 study, described previously (ClinicalTrials.gov, NCT01064856), ${ }^{2}$ was a phase III, multicentre, randomised, double-blind, placebo-controlled trial in patients with active non-psoriatic pSpA. Patients were randomised 1:1 to receive adalimumab (AbbVie, North Chicago, Illinois, USA) $40 \mathrm{mg}$ every other week or placebo during a 12-week double-blind period, ${ }^{2}$ followed by an open-label period of up to 3 years of adalimumab treatment. Eligible patients were aged $\geq 18$ years, fulfilled the ASAS criteria for $\mathrm{pSpA}^{1}$ and had pSpA symptoms for $\geq 3$ months before the study baseline visit. Patients had active disease, a score $\geq 40 \mathrm{~mm}$ on a $0-100 \mathrm{~mm}$ visual analogue scale (VAS) for Patient Global Assessment of disease activity (PtGA) and Patient Global Assessment of pain (PtGA-pain) and an inadequate response to $\geq 2$ non-steroidal anti-inflammatory drugs (NSAIDs) or intolerance to or contraindication for NSAIDs. The study was performed in accordance with the International Conference on Harmonisation Guidelines for Good Clinical Practice and the Declaration of Helsinki.

\section{Outcome measures}

Patients were seen at baseline and weeks 2, 4, 8 and 12 of the double-blind study, then every 4 weeks until week 28 , then every 8 weeks until week 68 and every 12 weeks thereafter. Disease activity outcome measures included PtGA, PtGA-pain, Physician Global Assessment (PGA), the Bath Ankylosing Spondylitis Disease Activity Index (BASDAI),${ }^{17}$ the Ankylosing Spondylitis Disease Activity Score (ASDAS) ${ }^{18}$ the Peripheral SpA Response Criteria (PSpARC; including PSpARC 20/40/50/70, defined as $\geq 20 \%, \geq 40 \%, \geq 50 \%$ or $\geq 70 \%$ improvement $(\geq 10, \geq 20$, $\geq 20$ or $\geq 30 \mathrm{~mm}$ absolute improvement on a VAS), respectively, from baseline in PtGA and PtGA-pain and $\geq 20 \%$, $\geq 40 \%, \geq 50 \%$ or $\geq 70 \%$ improvement from baseline in $\geq 1$ of the following: tender joint count (TJC)/swollen joint count (SJC), total enthesitis count or dactylitis count $)^{2}$ and C-reactive protein (CRP). The ASDAS was calculated as described previously $(0.12 \times$ back pain $+0.06 \times$ duration of morning stiffness $+0.11 \times \mathrm{PtGA}+0.07 \times$ peripheral pain/ swelling $+0.58 \times($ natural logarithm of CRP +1$)) .{ }^{18} 19$ Two separate remission criteria were evaluated. PSpARC remission was defined as $\mathrm{SJC} \leq 1$ plus $\geq 4$ of the following five: PtGA $\leq 20 \mathrm{~mm}$ VAS; PtGA-pain $\leq 20 \mathrm{~mm}$ VAS; TJC $\leq 1$; enthesitis count $\leq 1$ and/or dactylitis count $\leq 1 .{ }^{2}$ Remission was also defined using ASDAS inactive disease (ASDAS ID; ASDAS score $<1.3) .{ }^{19}$

Sustained remission was defined as achieving the specified remission criterion (PSpARC or ASDAS ID) for $\geq 24$ consecutive weeks ${ }^{811} 1620$ at any time during the 3-year study. The number of patients who achieved sustained remission in the first, second and third year of adalimumab exposure was calculated. In addition, we assessed how many patients experienced a disease flare after achieving sustained remission. For patients who achieved sustained ASDAS ID, flare was defined as $\geq 2$ consecutive study visits with ASDAS $\geq 2.1$ and/ or $\geq 1$ visit with ASDAS $>3.5 .^{21}{ }^{22}$ For patients who achieved sustained PSpARC remission, flare was defined as not 
A
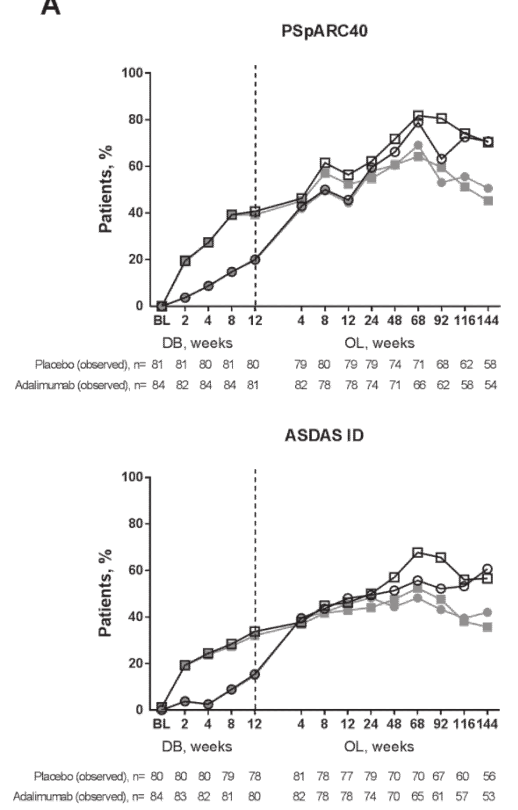

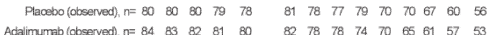
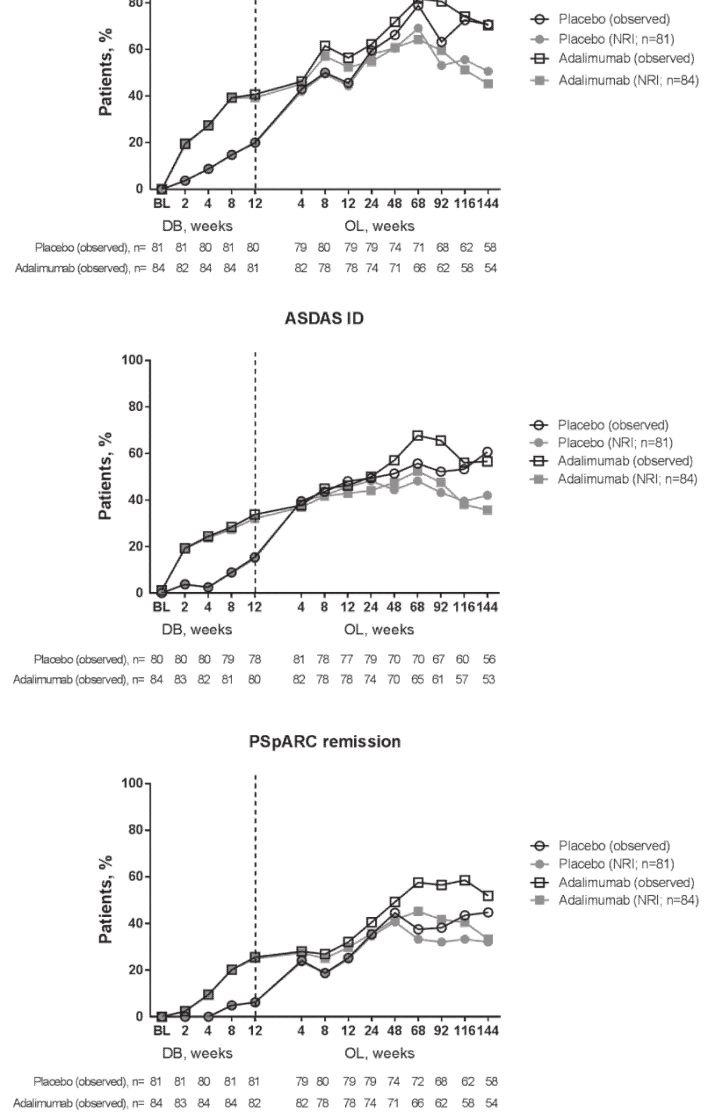

B

ASDAS
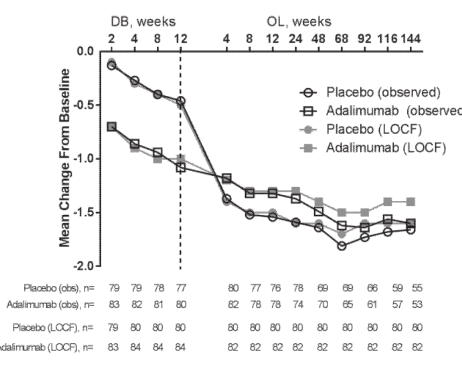

PtGA

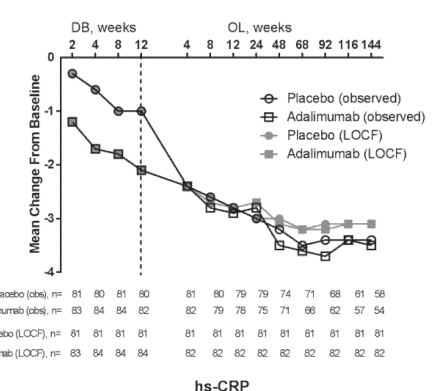

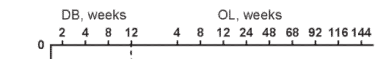
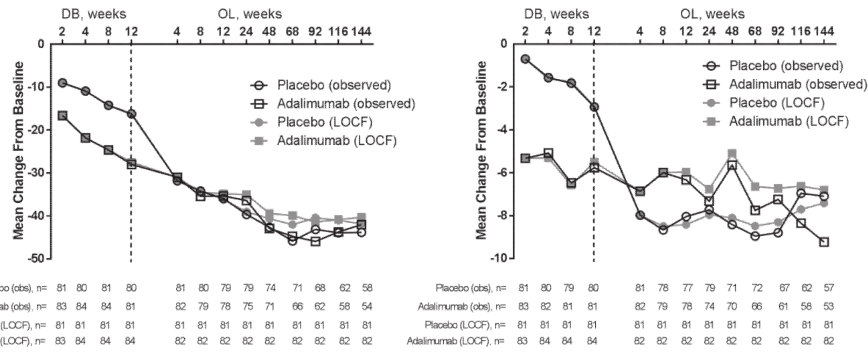

Figure 1 Percentage of patients achieving clinical outcomes (A) and mean changes from baseline (B). Clinical outcomes included (A) PSpARC40, ASDAS ID and PSpARC remission achievement and (B) ASDAS, BASDAI, PtGA and hs-CRP mean changes over 144 weeks in patients treated with adalimumab and placebo (up to week 12). Patients randomised to receive placebo during the DB period initiated OL adalimumab after week 12. ASDAS, Ankylosing Spondylitis Disease Activity Score; ASDAS ID, ASDAS inactive disease; BASDAI, Bath Ankylosing Spondylitis Disease Activity Index; BL, baseline; DB, double blind; hs-CRP, high-sensitivity C-reactive protein; LOCF, last observation carried forward; obs, observed; OL, open label; PtGA, Patient Global Assessment; PSpARC, Peripheral SpondyloArthritis Response Criteria.

achieving PSpARC40 (PSpARC40-non-responder) at $\geq 2$ consecutive visits.

Three different enthesitis tools were used in this study (the Maastricht Ankylosing Spondylitis Enthesitis Score, the Leeds Enthesitis Index and the Spondyloarthritis Research Consortium of Canada Enthesitis Index); to calculate the score using these three tools, a total of 29 different entheseal sites were examined per visit and patient. $^{2}$

\section{Statistical methods}

Descriptive statistics were used to report mean change from baseline (as observed and last observation carried forward analyses) in ASDAS, BASDAI, PtGA and high-sensitivity CRP (hs-CRP) as well as the percentages of patients (as observed and using non-responder imputation (NRI)) achieving PSpARC remission, ASDAS ID and PSpARC40 per visit over 144 weeks assessed by initial randomisation to adalimumab or placebo.

\section{Univariate analysis}

Univariate logistic regression was used to evaluate predictors of remission (PSpARC remission and ASDAS ID) at years 1 and 3. Possible predictors for remission after 1 and 3 years of adalimumab treatment were selected a priori; demographic and baseline disease characteristics included age, sex, body mass index (BMI), smoking status (current smoker vs current non-smoker), symptom duration, human leucocyte antigen B27-positive status, elevated baseline hs-CRP, prior use of disease-modifying antirheumatic drugs (DMARDs) and baseline values of the following: TJC, SJC, enthesitis count, BASDAI, ASDAS, PtGA and PGA. The response at week 12 of adalimumab 
A

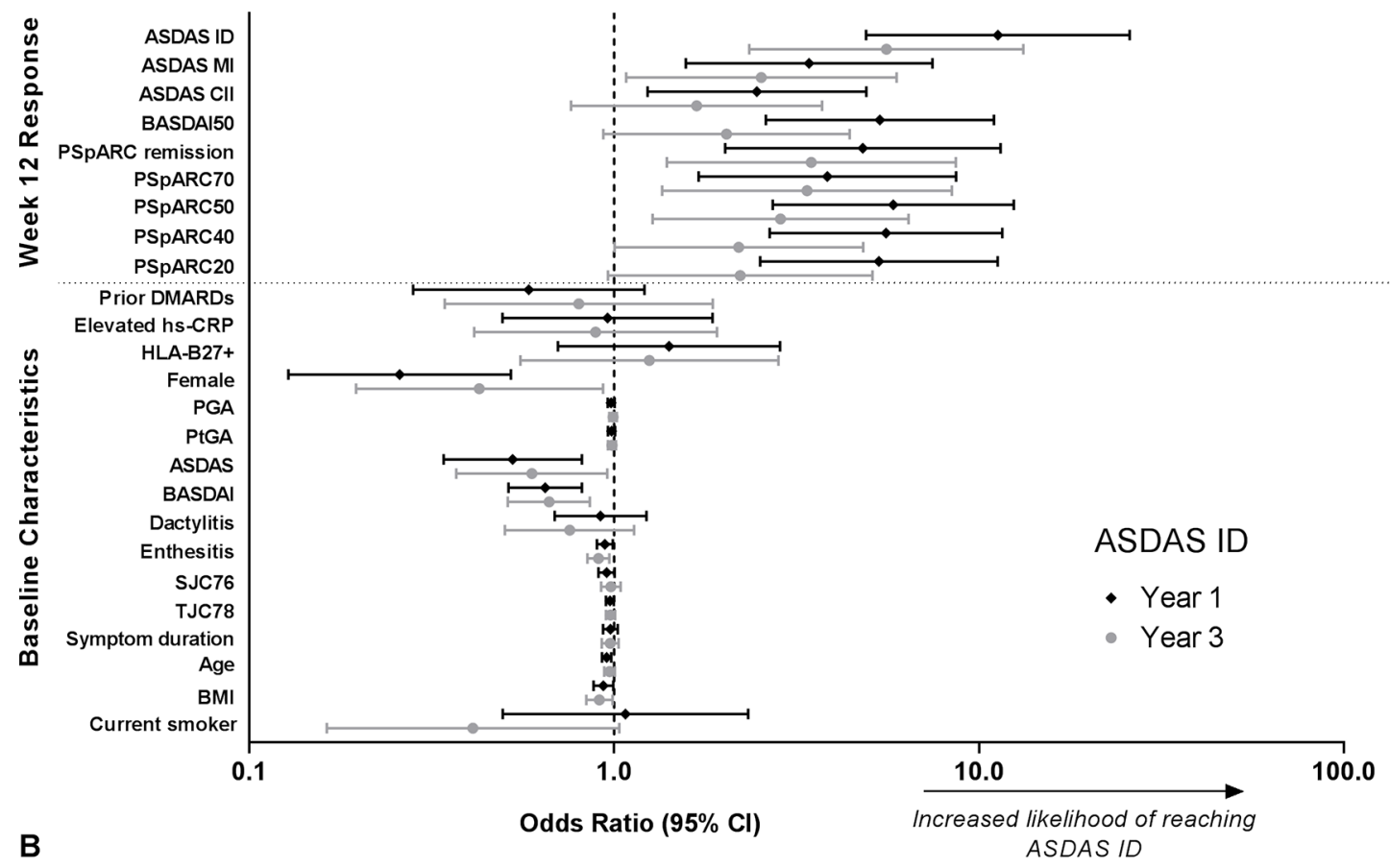

B

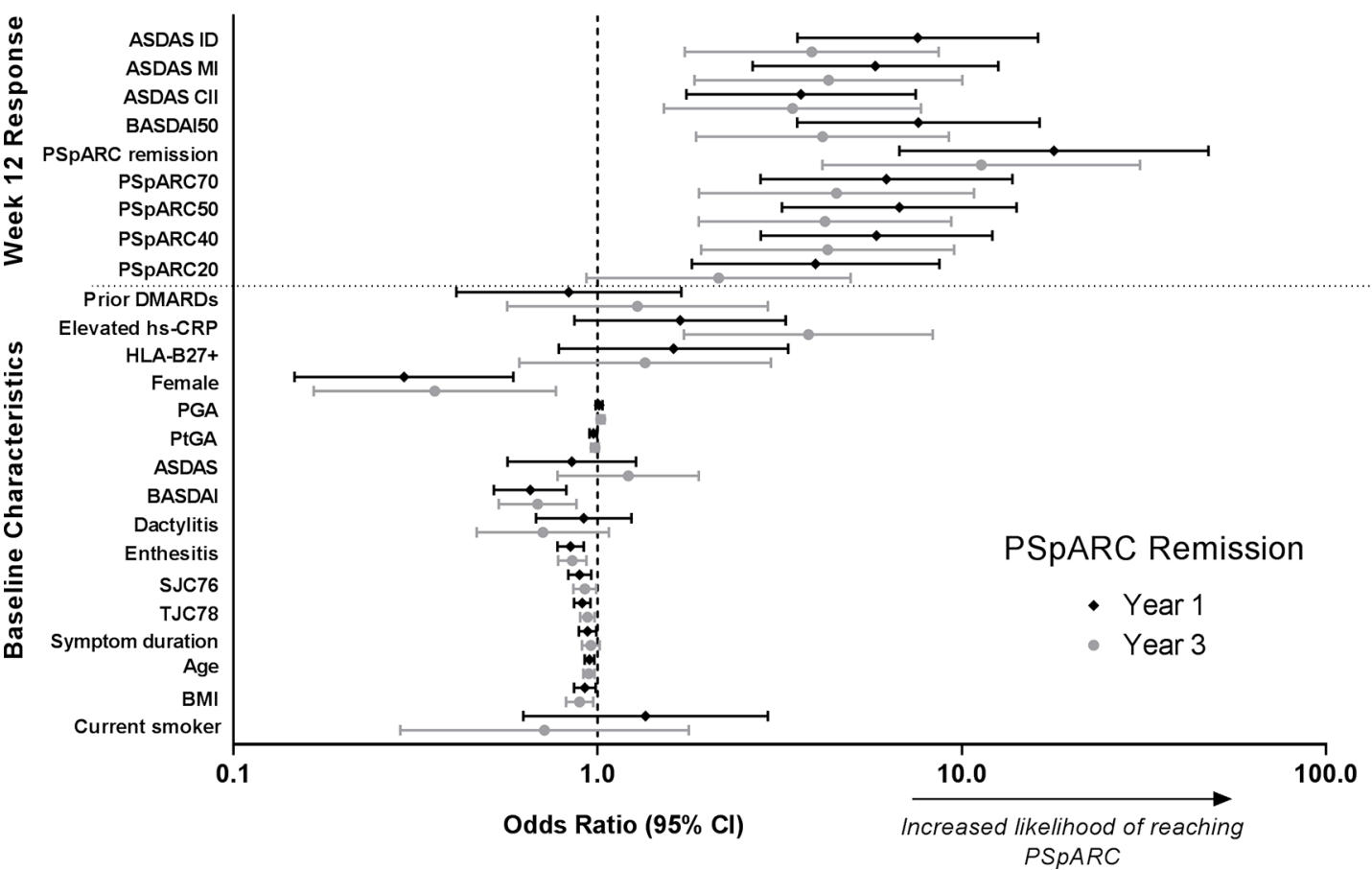

Figure 2 Univariate predictors of (A) ASDAS ID $(<1.3)$ and (B) PSpARC remission. Predictors of remission shown after 1 and 3 years of adalimumab exposure. ASDAS, Ankylosing Spondylitis Disease Activity Score; ASDAS CII, ASDAS clinically important improvement; ASDAS ID, ASDAS inactive disease; ASDAS MI, ASDAS major improvement; BASDAI, Bath Ankylosing Spondylitis Disease Activity Index; BMI, body mass index; DMARD, disease-modifying antirheumatic drug; HLA, human leucocyte antigen; hs-CRP, high-sensitivity C-reactive protein; PGA, Physician Global Assessment; PtGA, Patient Global Assessment; PSpARC, Peripheral SpondyloArthritis Response Criteria; SJC, swollen joint count; TJC, tender joint count.

exposure was assessed using $\geq 50 \%$ improvement in BASDAI (BASDAI50), ASDAS clinically important improvement (ASDAS CII; $\Delta \geq 1.1$ ), ASDAS major improvement (ASDAS MI; $\Delta \geq 2.0$ ), ASDAS ID, PSpARC20, PSpARC40, PSpARC50, PSpARC70 and PSPARC remission.
Multivariate analysis

Multivariate analysis was performed to determine predictors of achieving sustained ASDAS ID or PSpARC remission at any time during the study. All 25 variables with a critical significance level of $10 \%$ in the univariate analysis were included in the multivariate analysis (full model). 
A
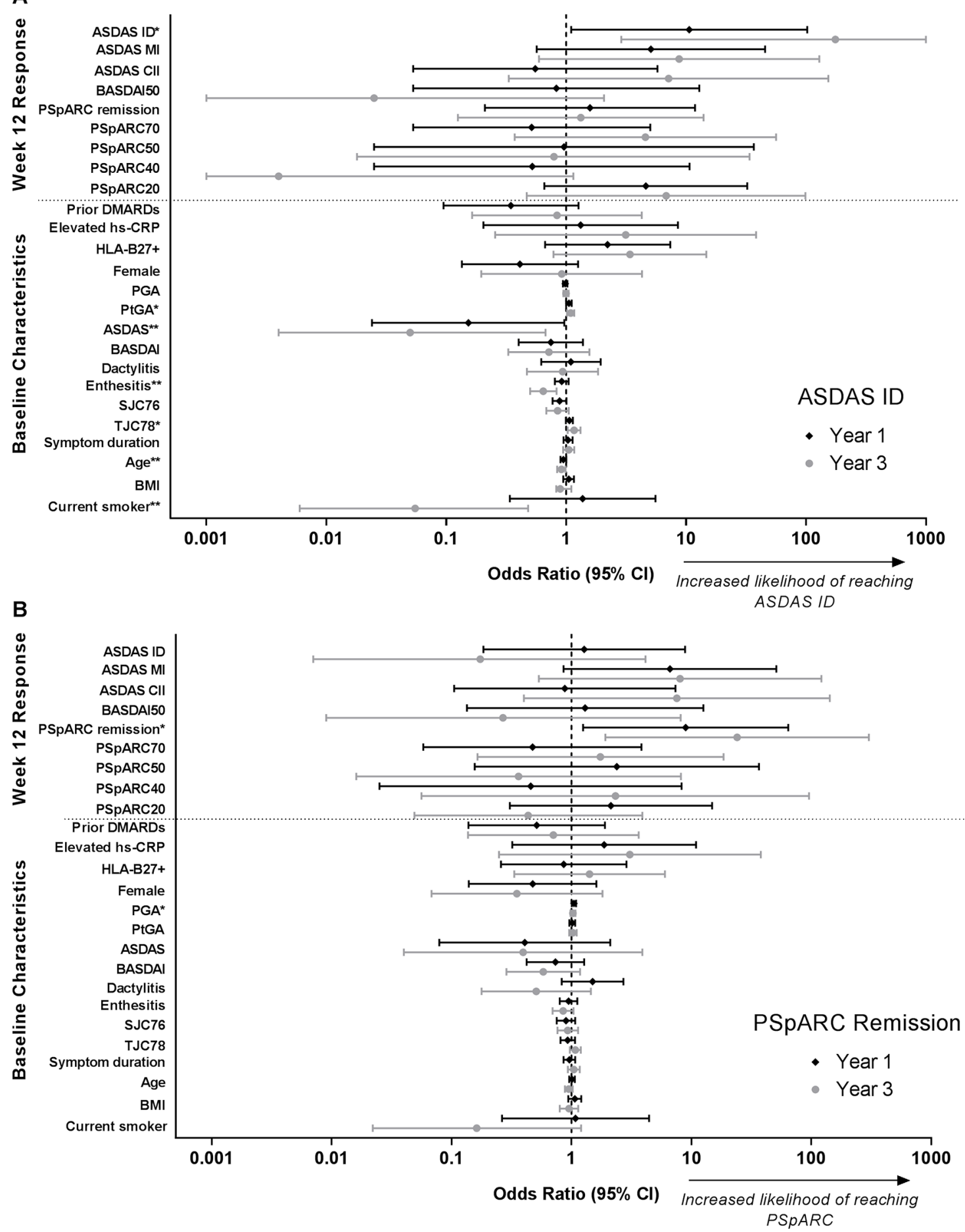

Figure 3 Multivariate predictors of (A) ASDAS ID (<1.3) and (B) PSpARC remission. Predictors of remission shown after 1 and 3 years of adalimumab exposure. ${ }^{*}$ Statistically significant positive predictor of remission $\left(\mathrm{P}<0.05 ; \chi^{2}\right.$ test). For $(\mathrm{A})$, includes ASDAS ID at week 12 (years 1 and 3) and PtGA and TJC (year 3); for (B), includes PSpARC remission (years 1 and 3) and PGA (year 1). ${ }^{* *}$ Statistically significant negative predictor of remission $\left(\mathrm{P}<0.05 ; \chi^{2}\right.$ test). For $(\mathrm{A})$, includes baseline ASDAS (years 1 and 3) and age, current smoker and enthesitis (year 3). ASDAS, Ankylosing Spondylitis Disease Activity Score; ASDAS CII, ASDAS clinically important improvement; ASDAS ID, ASDAS inactive disease; ASDAS MI, ASDAS major improvement; BASDAI, Bath Ankylosing Spondylitis Disease Activity Index; BMI, body mass index; DMARD, disease-modifying antirheumatic drug; HLA, human leucocyte antigen; hs-CRP, high-sensitivity C-reactive protein; PGA, Physician Global Assessment; PtGA, Patient Global Assessment; PSpARC, Peripheral SpondyloArthritis Response Criteria; SJC, swollen joint count; TJC, tender joint count. 
Table 2 Significant predictors of sustained remission over 3 years of adalimumab treatment

\begin{tabular}{|c|c|c|c|c|}
\hline \multirow[b]{2}{*}{ Predictor, OR (95\% CI) } & \multicolumn{2}{|c|}{ Sustained ASDAS ID } & \multicolumn{2}{|c|}{ Sustained PSpARC remission } \\
\hline & Full model & Reduced model & Full model & Reduced model \\
\hline \multicolumn{5}{|c|}{ Baseline demographic and disease characteristics } \\
\hline Normal hs-CRP (no vs yes) & NS & 0.18 (0.06 to 0.52$)$ & NS & NS \\
\hline Enthesitis & NS & NS & 0.79 (0.68 to 0.93$)$ & 0.85 (0.77 to 0.95$)$ \\
\hline Prior DMARDs (yes vs no) & NS & NS & $0.20(0.05$ to 0.74$)$ & $0.22(0.07$ to 0.66$)$ \\
\hline BASDAI & NS & $0.57(0.41$ to 0.78$)$ & NS & $0.70(0.50$ to 0.97$)$ \\
\hline $\mathrm{BMI}$ & NS & NS & NS & 0.88 (0.77 to 0.99$)$ \\
\hline \multicolumn{5}{|l|}{ Treatment response (yes vs no) } \\
\hline ASDAS MI at week 12 & 8.19 (1.08 to 61.91$)$ & NS & NS & NS \\
\hline ASDAS ID at week 12 & NS & 8.01 (2.47 to 25.97$)$ & NS & NS \\
\hline PSpARC remission at week 12 & NS & NS & 13.05 (1.75 to 97.52$)$ & 20.27 (5.37 to 76.46$)$ \\
\hline
\end{tabular}

ASDAS, Ankylosing Spondylitis Disease Activity Score; ASDAS ID, ASDAS inactive disease; ASDAS MI, ASDAS major improvement ( $\triangle \geq 2.0$ ); BASDAI, Bath Ankylosing Spondylitis Disease Activity Index; BMI, body mass index; DMARD, disease-modifying antirheumatic drug; hsCRP, high-sensitivity C-reactive protein; NS, not significant; PSpARC, Peripheral SpondyloArthritis Response Criteria.

To reduce the number of explanatory variables entering the analysis while maximising the variation explained by them, a backward variable selection in logistic regression analysis was conducted to obtain the best model (elimination model), which isolates the most influential contributing factors. ${ }^{23}$

All statistical analyses were conducted using SAS V.9.3 (SAS Institute, Cary, North Carolina, USA).

\section{RESULTS}

Patient disposition

A total of 165 patients were initially randomised to adalimumab $(n=84)$ or placebo $(n=81)$; in the open-label phase, all patients received $\geq 1$ dose of adalimumab. Approximately half of the patients were women and $44 \%$ had an elevated CRP at baseline. The mean (SD) age was 41 (12) years and symptom duration was 7 (7) years (table 1).

Of the 165 patients, $163(99 \%)$ completed the week 12 visit and $114(69 \%)$ completed the week 156 visit. The primary reasons for premature discontinuation were adverse events $(n=19)$, withdrawal of consent $(n=12)$, loss to follow-up $(n=3)$ or other reasons $(n=14$; including lack of efficacy, pregnancy, investigator decision and patient relocation).

\section{Adalimumab efficacy over 3 years}

The percentages of patients who achieved PSpARC40 (the primary endpoint of ABILITY-2), ${ }^{2}$ ASDAS ID and PSpARC remission increased over time, generally reaching a plateau around week 48 of the open-label period, in observed as well as NRI analyses (figure 1A). In the observed analysis, ASDAS ID was achieved by $55 \%(78 / 143)$ of patients at 1 year and $59 \%(64 / 109)$ of patients at 3 years; $41 \%(60 / 145)$ and $48 \%(54 / 112)$ achieved PSpARC remission, respectively. Using NRI analysis, $47 \%$ and $39 \%$ of patients achieved ASDAS ID and $36 \%$ and $33 \%$ achieved PSpARC remission at years 1 and 3, respectively. During the 3-year study, more patients achieved sustained ASDAS ID $(52 \%(86 / 165))$ than sustained PSpARC remission $(42 \%(70 / 165))$.

Improvements in the signs and symptoms of pSpA after 1 year of adalimumab treatment were sustained through 3 years. Continuous efficacy outcomes showed improvement as early as 2 weeks after initiation of adalimumab treatment, and improvements were sustained throughout the study in patients originally randomised to receive adalimumab in observed as well as last observation carried forward analyses (figure 1B). In patients originally randomised to receive placebo, similar findings were observed after initiation of open-label adalimumab at week 12.

Of the 86 patients who achieved sustained ASDAS ID, $64 \%(55 / 86)$ did so in the first year of adalimumab exposure, whereas only $7 \%(6 / 86)$ had delayed achievement in the third year of exposure. Only two patients (2\%) had a subsequent disease flare after achieving sustained ASDAS ID. Notably, of the 31 patients who did not achieve sustained ASDAS ID in the first year, most (87\%) achieved ASDAS ID on $\geq 1$ visit in the first year of adalimumab exposure.

Similarly, of the 70 patients who achieved sustained PSpARC remission, $60 \%(42 / 70)$ did so in the first year of adalimumab exposure, whereas only $7 \%(5 / 70)$ had a delayed onset of sustained PSpARC remission in the third year of exposure. Among patients who achieved sustained PSpARC remission, only seven patients (10\%) had a subsequent disease flare. Of the 28 patients who did not achieve sustained PSpARC remission in the first year, most $(82 \%)$ achieved PSpARC remission on $\geq 1$ visit in the first year of adalimumab exposure.

Additionally, there was a large overlap between patients who achieved sustained remission definitions: of 70 patients achieving sustained PSpARC remission, 63 (90\%) also achieved sustained ASDAS ID. 
Table 3 Treatment-emergent AEs

\begin{tabular}{lc}
\hline Event (Event/100 PY) & $\begin{array}{l}\text { Any adalimumab } \\
\mathbf{n = 1 6 5} \\
\mathbf{P Y s}=390.7\end{array}$ \\
\hline Any AE & $1194(305.6)$ \\
\hline Severe AE & $36(9.2)$ \\
\hline Serious AE & $29(7.4)$ \\
\hline AE leading to study discontinuation & $31(7.9)$ \\
Infectious AE & $376(96.2)$ \\
Serious infectious AE & $4(1.0)$ \\
\hline Opportunistic infections (excluding oral & $1(0.3)$ \\
candidiasis and tuberculosis) & \\
Tuberculosis* & $2(0.5)$ \\
Lymphoma & 0 \\
NMSC & $1(0.3)$ \\
\hline Malignancies (excluding lymphoma, & $2(0.5)$ \\
HSTCL, leukaemia, NMSC or & \\
melanoma) & \\
\hline Demyelinating disorder & 0 \\
\hline Deaths & $2(0.5)$ \\
\hline
\end{tabular}

${ }^{*}$ Category captured active and latent tuberculosis; however, only latent cases were reported.

AE, adverse event; HSTCL, hepatosplenic T-cell lymphoma; NMSC, non-melanoma skin cancer; PY, patient-year.

\section{Predictors of remission at years 1 and 3}

Univariate analysis showed that week 12 treatment response rates were more consistent predictors of remission at both years 1 and 3 compared with baseline disease characteristics. Significant positive predictors of ASDAS ID and PSpARC remission at years 1 and 3 were PSpARC remission as well as other PSpARC response categories at week 12 and ASDAS ID and other ASDAS response categories (figure 2A and 2B) at week 12. Of note, elevated baseline hs-CRP was a predictor of PSpARC remission at year 3 only and was not predictive of ASDAS ID at either year 1 or year 3 .

In multivariate analyses (full model), only ASDAS ID at week 12 remained as a consistent and strong predictor for ASDAS ID at years 1 (OR, 10.61 (95\% CI 1.10 to 102.25$)$ ) and 3 (OR, 175.79 (95\% CI 2.88 to $>999$ ); figure 3A). Higher baseline disease activity, measured by ASDAS, was associated with a lower chance of achieving ASDAS ID at years 1 and 3, while the baseline entheseal count was a negative predictor of ASDAS ID at year 3. Baseline TJC and PtGA were only marginally predictive of ASDAS ID response at year 3 (OR, 1.16 and 1.09, respectively).

In backward elimination modelling, ASDAS ID at week 12 was a consistent predictor of ASDAS ID at year 1 (OR, $8.64(95 \%$ CI 2.97 to 25.14$)$ ) and year 3 (OR, $36.12(95 \%$ CI 2.29 to 569.08) ), as was baseline ASDAS at year 1 (OR, $0.31(95 \%$ CI 0.16 to 0.62$)$ ) and at year 3 (OR, $0.10(95 \%$ CI 0.02 to 0.45$)$ ).

In multivariate analyses (full model), only PSpARC remission at week 12 remained as a consistent and strong predictor of PSpARC remission at both years 1 (OR, 8.96 (95\% CI 1.25 to 64.17)) and 3 (OR, 24.08 (95\% CI 1.92 to 302.21); figure 3B). Baseline PGA was marginally associated with PSpARC remission at year 1 (OR, 1.05 (95\% CI 1.00 to 1.09)). Notably, ASDAS ID at week 12 was not predictive of long-term PSpARC remission at years 1 and 3 , and PSpARC remission at week 12 was not predictive of ASDAS ID at years 1 and 3 .

In backward elimination modelling, PSpARC remission at week 12 was a consistent predictor of PSpARC remission at 1 year (OR, 6.47 (95\% CI 1.91 to 21.95$)$ ) and at 3 years (OR, 15.66 (95\% CI 4.19 to 58.56)).

\section{Predictors of sustained remission over 3 years of adalimumab treatment}

In backward elimination modelling, ASDAS ID at week 12 was a predictor of sustained ASDAS ID (table 2). PSpARC remission at week 12 was a consistent predictor of sustained PSpARC remission in all multivariate analyses, including the full model and the backward elimination model.

Only marginal associations with sustained remission were found for other factors (table 2). Baseline enthesitis count (full and elimination models), BASDAI (elimination model), BMI (elimination model) and prior use of DMARDs (full and elimination models) were negatively associated with achievement of sustained PSpARC remission. Baseline BASDAI and elevated hs-CRP were negative predictors of sustained ASDAS ID (elimination model).

\section{Long-term safety}

Patients had 390.7 patient-years of adalimumab exposure in this 3-year study. Rates of serious adverse events and serious infections were low (7.4 and 1.0 events per 100 patient-years, respectively; table 3 ). There were no cases of lymphoma, demyelinating disorders or active tuberculosis. Two patients died: a 20-year-old man died of injuries resulting from a car accident and a 58-year-old woman of pulmonary embolism. One event of opportunistic infection (bronchopulmonary aspergillosis) in a 47-year-old woman resolved with appropriate treatment. One case of parasitic infection (acarodermatitis) in a 43-year-old woman led to discontinuation from the study but resolved post-treatment. Two malignancies were reported: one patient had non-melanoma skin cancer and one patient had bilateral pheochromocytoma. The incident of pheochromocytoma was subsequently treated by laparoscopic bilateral adrenalectomy and resolved.

\section{DISCUSSION}

This is the first study in patients with pSpA evaluating the long-term efficacy and safety of a TNF inhibitor over 3 years and evaluating predictors of long-term remission. Most patients showed early and sustained improvements with adalimumab treatment. Among patients who continued study drug, approximately 50\% achieved sustained remission defined by ASDAS ID and approximately $40 \%$ when defined by PSpARC remission criteria, 
most within the first year of adalimumab exposure without subsequent flare. Consistent and strong predictors for long-term (1 and 3 years) ASDAS ID and PSpARC remission as well as sustained ASDAS ID and PSpARC remission were early (week 12) responses for ASDAS ID and PSpARC remission.

The long-term and sustained remission rates from our study are consistent with other studies in patients with $\mathrm{RA},{ }^{8}{ }^{1124} \mathrm{AS}^{16}$ and $\mathrm{PsA}^{15}$ treated with various therapies, including TNF inhibitors and DMARDs. Additionally, within our study, sustained remission rates were similar to point remission rates at 1 and 3 years, emphasising the durability of response to adalimumab in this population.

In some studies of patients with $\mathrm{RA}^{9}$ and $\mathrm{AS},{ }^{25-28}$ baseline demographic characteristics, such as younger age or elevated CRP, predicted short-term response and remission. In our study, no baseline variables, including BMI, smoking status, hs-CRP and sex, consistently predicted long-term or sustained response. However, we examined predictors of long-term rather than short-term remission. Similar to our study, multiple studies have found that good early clinical response after treatment initiation predicts good long-term outcomes for up to 1 year or longer. ${ }^{71011162930}$ For example, in a similarly designed randomised, double-blind, placebo-controlled phase III study in patients with AS receiving adalimumab, achievement of early (12-week) remission was a stronger predictor of favourable long-term (up to 5 years) treatment outcomes than baseline characteristics, including elevated CRP. ${ }^{16}$ In axial SpA and PsA, the lack of a robust early (12-week) clinical response to another anti-TNF treatment, certolizumab, identified a subset of patients who were unlikely to achieve a good response at 48 weeks. ${ }^{31}$ Defining early clinical response as 12 weeks after initiation of new treatment aligns with studies and treatment recommendations in RA and axial SpA. ${ }^{32} 33$

The presence or absence of early response to treatment can also impact long-term outcomes in other non-SpA rheumatic diseases. For example, in a study of patients with very early RA, worse outcomes (greater radiographic progression, Health Assessment Questionnaire disability and number of workdays missed) were observed for patients who had persistent moderate disease activity versus those who achieved early sustained clinical remission during the first year. ${ }^{24}$ Also, associations between early response and long-term remission have been reported in multiple other studies in RA, ${ }^{71329}$ and earlier time to remission was a consistent and strong predictor of sustained remission in patients with RA. ${ }^{811}$

As mentioned above, baseline patient factors were less robustly predictive for long-term response in our study. Other studies have reported an association of female sex with a decreased likelihood of achieving remission. ${ }^{1134}$ In our study, female sex was a negative predictor for achieving ASDAS ID and PSpARC remission at 1 and 3 years in univariate analyses only (figure $2 \mathrm{~A}$ and $\mathrm{B}$ ), but this finding was not confirmed in the more stringent multivariate analysis (figure $3 \mathrm{~A}$ and B). Similarly, tobacco use was a negative predictor of ASDAS ID at year 3 only, and BMI only marginally predicted sustained PSpARC remission but not PSpARC remission at 1 year or 3 years or ASDAS ID at 1 year, 3 years or sustained ASDAS ID. Both smoking and obesity have been described as negative predictors of clinical response in some RA, axial SpA and PsA studies ${ }^{1034-37}$ but not in others ${ }^{913} 343839$; future studies are needed to confirm these results, especially in patients with $\mathrm{pSpA}$. Baseline PGA was also marginally predictive of PSpARC remission at 1 year in our full model. Although the ORs for some measurements from our full models had large CIs, the direction of the treatment effects was confirmed by findings in the reduced models. Intuitively, patients with lower baseline values for disease activity measures would seem better positioned to achieve a low disease activity state in RA, and studies support this expectation. ${ }^{12} 34{ }^{40}$ In our multivariate and backward elimination analyses, baseline ASDAS predicted ASDAS ID at years 1 and 3, and baseline BASDAI predicted sustained ASDAS ID and PSpARC in the reduced models.

In this study, two different definitions of remission were used. PSpARC remission, which differs from ASDAS ID in that it includes aspects of the disease such as TJC and SJC and enthesitis, appears to be more suitable for this patient population given its better face validity. The different domains captured by ASDAS and PSpARC response might explain why ASDAS ID at week 12 was not predictive of long-term PSpARC remission and vice versa. Although ASDAS was established in patients with AS, a recent analysis showed that AS-specific outcome measures (including ASDAS) perform reasonably well in $\mathrm{pSpA}$ in terms of discrimination. ${ }^{41}$ Despite conceptual differences, the overlap between patients achieving sustained ASDAS ID and PSpARC remission was considerable. Also, minimal disease activity, which has been used in PsA and captures very similar disease aspects as PSpARC, ${ }^{42}$ has recently been evaluated in this patient population. $^{43}$

There is no consensus on the length of time required for remission to be considered sustained in $\mathrm{pSpA}$; however, this is also true in other rheumatic diseases, including RA, for which the 2016 European League Against Rheumatism treatment recommendations specify a treatment goal of sustained remission or low disease activity. ${ }^{13} 4445$ The defined timing for achieving sustained remission in $\mathrm{pSpA}$ used in our analysis ( $\geq 6$ months out of 3 years) was based on other published studies in RA and axial SpA. ${ }^{81620}$ Although some studies in patients with RA used up to 5 or 8 years to define sustained remission, ${ }^{12}{ }^{44}$ and other RA or PsA studies used 1 year, ${ }^{10}{ }^{15}$ those were mostly cohort studies and not randomised controlled trials, with widely spaced visit intervals (every 6-12 months or longer), unlike the prescribed shorter intervals in our study. Nonetheless, defining sustained remission as $\geq 6$ months may not adequately reflect patients' clinical condition over the course of 3 years of follow-up and may be considered a study limitation. Still, our chosen 
definition of sustained remission appears to have clinical utility because patients who achieved sustained remission, whether defined by ASDAS ID or PSpARC, rarely experienced subsequent disease flare (rate $\leq 10 \%$ ).

Identification of factors that predict long-term and sustained remission in patients with $\mathrm{pSpA}$ after treatment with anti-TNF agents may facilitate clinical decisions regarding patient therapy. Because our study did not include imaging assessments, future research can help to establish whether a state of sustained remission might prohibit structural damage progression, as has been shown in RA and PsA. ${ }^{15} 244647$ Additional investigation might also address whether patients who achieve consistent sustained remission would benefit from drug withdrawal; so far, drug-free remission appears feasible in only a limited subset of patients with early RA and axial SpA. $^{48-50}$

Most patients in our study achieved sustained remission within the first year of treatment with adalimumab. In patients with RA, shorter time to remission has been associated with sustainability of remission. ${ }^{811}$ Among patients with $\mathrm{pSpA}$ who achieved sustained remission in the second or third year of adalimumab treatment, a substantial proportion met remission criteria at individual but not consecutive visits in the first year. Sustained remission is a more stringent endpoint; patients who achieve sustained remission after a delay show good response at earlier time points. Clinically, this also suggests continuing therapy may be warranted in patients who do not achieve (sustained) remission during the first year owing to some fluctuations in disease activity.

The safety findings, including the type and frequency of adverse events observed through 3 years of adalimumab treatment, are in line with the known overall safety profile of adalimumab in other immune-mediated inflammatory diseases. ${ }^{51}{ }^{52}$ The serious infection rate observed in our study (1.0 event per 100 patient-years) was numerically lower than for RA and PsA but similar to AS. ${ }^{53}$ These findings need further confirmation.

Overall, among participants in the ABILITY-2 study receiving adalimumab, most achieved and maintained long-term efficacy, a significant number achieved sustained remission without subsequent flare and a rapid response after initiation of adalimumab treatment was the best predictor of sustained remission over time.

\section{Author affiliations \\ ${ }^{1}$ Department of Rheumatology, Ghent University Hospital, Ghent, Belgium ${ }^{2}$ Swedish Medical Center and University of Washington, Seattle, Washington, USA ${ }^{3}$ Department of Gastroenterology, Infectiology, and Rheumatology, Charité Universitätsmedizin Berlin, Berlin, Germany \\ ${ }^{4}$ Department of Experimental Immunology, Academic Medical Centre, University of Amsterdam, Amsterdam, The Netherlands \\ ${ }^{5}$ Department of Medicine, University of Illinois at Chicago, Chicago, Illinois, USA \\ ${ }^{6}$ Data and Statistical Sciences, AbbVie Inc., North Chicago, Illinois, USA \\ ${ }^{7}$ Immunology Clinical Development, AbbVie Inc., North Chicago, Illinois, USA}

Acknowledgements The authors thank Nupun A Varothai, $\mathrm{PhD}$, formerly of AbbVie, for his contributions to the statistical analysis and manuscript. Medical writing support was provided by Katie Singh, PhD and Maria Hovenden, PhD, of
Complete Publication Solutions, LLC (North Wales, Pennsylvania, USA). AbbVie funded the research, statistical and medical writing support.

Contributors The authors and AbbVie scientists designed the study and analysed and interpreted the data. All authors contributed to the development of the content; all authors and AbbVie reviewed and approved the manuscript; the authors maintained control over the final content.

Funding This work was supported by AbbVie Inc.

Competing interests $\mathrm{FVdB}$ has received research grants, consulting fees and speaker's fees from AbbVie, Celgene, Janssen, Merck, Novartis, Pfizer and UCB. PJM has received research grants, consulting fees and/or speaker's fees from AbbVie, Amgen, Boehringer Ingelheim, Bristol-Myers Squibb, Celgene, Genentech, Janssen, Lilly, Merck, Novartis, Pfizer, Sun and UCB. JS has received research grants, consulting fees and speaker's fees from AbbVie, Merck, Pfizer and UCB. DLB is a part-time employee of UCB and has received funding from AbbVie, Pfizer, MSD, UCB, Janssen, Novartis, Eli Lilly, Boehringer Ingelheim, BMS, Roche and Glenmark. YX is a former employee of AbbVie and may own AbbVie stock and/ or options. SC, ALP and I-HS are employees of AbbVie and may own AbbVie stock and/or options.

Ethics approval The study was approved by the ethics review board of the main institution (Western Institutional Review Board; date of approval: 20 May 2010; ethics approval/site number: $4935 \mathrm{~W}-10$ ) and ethics review boards of each additional centre that participated in the study.

Provenance and peer review Not commissioned; externally peer reviewed.

Open Access This is an Open Access article distributed in accordance with the Creative Commons Attribution Non Commercial (CC BY-NC 4.0) license, which permits others to distribute, remix, adapt, build upon this work non-commercially, and license their derivative works on different terms, provided the original work is properly cited and the use is non-commercial. See: http://creativecommons.org/ licenses/by-nc/4.0/

(C) Article author(s) (or their employer(s) unless otherwise stated in the text of the article) 2018. All rights reserved. No commercial use is permitted unless otherwise expressly granted.

\section{REFERENCES}

1. Rudwaleit M, van der Heijde D, Landewé R, et al. The Assessment of SpondyloArthritis International Society classification criteria for peripheral spondyloarthritis and for spondyloarthritis in general. Ann Rheum Dis 2011;70:25-31.

2. Mease P, Sieper J, Van den Bosch F, et al. Randomized controlled trial of adalimumab in patients with nonpsoriatic peripheral spondyloarthritis. Arthritis Rheumatol 2015;67:914-23.

3. Van Den Bosch F, Kruithof E, Baeten D, et al. Randomized doubleblind comparison of chimeric monoclonal antibody to tumor necrosis factor alpha (infliximab) versus placebo in active spondylarthropathy. Arthritis Rheum 2002;46:755-65.

4. Paramarta JE, De Rycke L, Heijda TF, et al. Efficacy and safety of adalimumab for the treatment of peripheral arthritis in spondyloarthritis patients without ankylosing spondylitis or psoriatic arthritis. Ann Rheum Dis 2013;72:1793-9.

5. Paramarta JE, Heijda TF, Baeten DL. Fast relapse upon discontinuation of tumour necrosis factor blocking therapy in patients with peripheral spondyloarthritis. Ann Rheum Dis 2013;72:1581-2.

6. Dougados M, van der Linden S, Juhlin R, et al. The European Spondylarthropathy Study Group preliminary criteria for the classification of spondylarthropathy. Arthritis Rheum 1991;34:1218-27.

7. Verschueren P, Esselens G, Westhovens R. Predictors of remission, normalized physical function, and changes in the working situation during follow-up of patients with early rheumatoid arthritis: an observational study. Scand J Rheumatol 2009;38:166-72.

8. Schipper LG, Fransen J, den Broeder AA, et al. Time to achieve remission determines time to be in remission. Arthritis Res Ther 2010;12:R97.

9. Pers YM, Fortunet C, Constant E, et al. Predictors of response and remission in a large cohort of rheumatoid arthritis patients treated with tocilizumab in clinical practice. Rheumatology 2014;53:76-84.

10. Navarro-Millán I, Chen L, Greenberg JD, et al. Predictors and persistence of new-onset clinical remission in rheumatoid arthritis patients. Semin Arthritis Rheum 2013;43:137-43.

11. Kuriya $\mathrm{B}$, Xiong $\mathrm{J}$, Boire $\mathrm{G}$, et al. Earlier time to remission predicts sustained clinical remission in early rheumatoid arthritis--results from the Canadian Early Arthritis Cohort (CATCH). J Rheumatol 2014;41:2161-6. 
12. Jayakumar K, Norton S, Dixey J, et al. Sustained clinical remission in rheumatoid arthritis: prevalence and prognostic factors in an inception cohort of patients treated with conventional DMARDs. Rheumatology 2012;51:169-75.

13. Barnabe C, Homik J, Barr SG, et al. The effect of different remission definitions on identification of predictors of both point and sustained remission in rheumatoid arthritis treated with anti-TNF therapy. $J$ Rheumatol 2014;41:1607-13.

14. Theander E, Husmark T, Alenius GM, et al. Early psoriatic arthritis: short symptom duration, male gender and preserved physical functioning at presentation predict favourable outcome at 5-year follow-up. Results from the Swedish Early Psoriatic Arthritis Register (SwePsA). Ann Rheum Dis 2014;73:407-13.

15. Coates LC, Cook R, Lee KA, et al. Frequency, predictors, and prognosis of sustained minimal disease activity in an observational psoriatic arthritis cohort. Arthritis Care Res 2010;62:970-6.

16. Sieper J, van der Heijde D, Dougados M, et al. Early response to adalimumab predicts long-term remission through 5 years of treatment in patients with ankylosing spondylitis. Ann Rheum Dis 2012;71:700-6.

17. Garrett S, Jenkinson T, Kennedy LG, et al. A new approach to defining disease status in ankylosing spondylitis: the Bath Ankylosing Spondylitis Disease Activity Index. J Rheumatol 1994;21:2286-91.

18. Lukas C, Landewé R, Sieper J, et al. Development of an ASASendorsed disease activity score (ASDAS) in patients with ankylosing spondylitis. Ann Rheum Dis 2009;68:18-24.

19. Machado P, Landewé R, Lie E, et al. Ankylosing Spondylitis Disease Activity Score (ASDAS): defining cut-off values for disease activity states and improvement scores. Ann Rheum Dis 2011;70:47-53.

20. Dougados M, van der Heijde D, Sieper J, et al. Clinical and imaging efficacy of etanercept in early non-radiographic axia spondyloarthritis: 104-week treatment results [abstract]. Arthritis Rheumatol 2015;67:713.

21. Study to evaluate maintenance of sustained remission of axSpA with CZP compared to placebo (C-OPTIMISE) (NCT02505542). https:// clinicaltrials.gov/ct2/show/NCT02505542 (accessed 28 Dec 2015).

22. Landewé R, Sieper J, Mease PJ, et al. Efficacy and safety of continuing versus withdrawing adalimumab in maintaining remission in patients with non-radiographic axial spondyloarthritis [abstract]. Arthritis Rheumatol 2017;69(Suppl 10):1787.

23. Royston P, Sauerbrei W. Selection of variables. Multivariable modelbuilding a pragmatic approach to regression analysis based on fractional polynomials for modelling continuous variables. Chichester, UK: John Wiley \& Sons, Ltd, 2008.

24. Combe B, Logeart I, Belkacemi MC, et al. Comparison of the longterm outcome for patients with rheumatoid arthritis with persistent moderate disease activity or disease remission during the first year after diagnosis: data from the ESPOIR cohort. Ann Rheum Dis 2015;74:724-9.

25. Arends S, Brouwer E, van der Veer E, et al. Baseline predictors of response and discontinuation of tumor necrosis factoralpha blocking therapy in ankylosing spondylitis: a prospective longitudinal observational cohort study. Arthritis Res Ther 2011;13:R94.

26. Vastesaeger $\mathrm{N}$, van der Heijde $\mathrm{D}$, Inman RD, et al. Predicting the outcome of ankylosing spondylitis therapy. Ann Rheum Dis 2011;70:973-81.

27. Glintborg B, Ostergaard M, Krogh NS, et al. Predictors of treatment response and drug continuation in 842 patients with ankylosing spondylitis treated with anti-tumour necrosis factor: results from 8 years' surveillance in the Danish nationwide DANBIO registry. Ann Rheum Dis 2010;69:2002-8.

28. Rudwaleit M, Claudepierre P, Wordsworth P, et al. Effectiveness, safety, and predictors of good clinical response in 1250 patients treated with adalimumab for active ankylosing spondylitis. $J$ Rheumatol 2009;36:801-8.

29. Aletaha D, Funovits J, Keystone EC, et al. Disease activity early in the course of treatment predicts response to therapy after one year in rheumatoid arthritis patients. Arthritis Rheum 2007;56:3226-35.

30. Keystone EC, Curtis JR, Fleischmann RM, et al. Rapid improvement in the signs and symptoms of rheumatoid arthritis following certolizumab pegol treatment predicts better longterm outcomes: post-hoc analysis of a randomized controlled trial. J Rheumatol 2011;38:990-6.

31. van der Heijde D, Deodhar A, Fleischmann R, et al. Early disease activity or clinical response as predictors of long-term outcomes with certolizumab pegol in axial spondyloarthritis or psoriatic arthritis. Arthritis Care Res 2017;69:1030-9.

32. van der Heijde D, Sieper J, Maksymowych WP, et al. 2010 Update of the international ASAS recommendations for the use of anti-
TNF agents in patients with axial spondyloarthritis. Ann Rheum Dis 2011;70:905-8.

33. Aletaha D, Alasti F, Smolen JS. Optimisation of a treat-to-target approach in rheumatoid arthritis: strategies for the 3-month time point. Ann Rheum Dis 2016;75:1479-85.

34. Katchamart W, Johnson S, Lin HJ, et al. Predictors for remission in rheumatoid arthritis patients: a systematic review. Arthritis Care Res 2010;62:1128-43.

35. Højgaard P, Glintborg B, Hetland ML, et al. Association between tobacco smoking and response to tumour necrosis factor $\otimes$ inhibitor treatment in psoriatic arthritis: results from the DANBIO registry. Ann Rheum Dis 2015;74:2130-6.

36. Eder L, Thavaneswaran A, Chandran V, et al. Obesity is associated with a lower probability of achieving sustained minimal disease activity state among patients with psoriatic arthritis. Ann Rheum Dis 2015;74:813-7.

37. Micheroli R, Hebeisen M, Wildi LM, et al. Impact of obesity on the response to tumor necrosis factor inhibitors in axia spondyloarthritis. Arthritis Res Ther 2017;19:164.

38. Smolen JS, Wang X, Sainsbury I, et al. Predicting DAS28-CRP $<2.6$ and low disease activity status at 6 months based on early clinical response in rheumatoid arthritis patients. Ann Rheum Dis 2016;75:187-8

39. Mease P, Poddubnyy D, Chen S, et al. Therapeutic response in adalimumab-treated patients with non-radiographic axial spondyloarthritis is similar regardless of body mass index. Arthritis Rheum 2014;66(Suppl 11):S245.

40. Mohammed $\mathrm{RH}$, Farahat $\mathrm{F}$, Kewan $\mathrm{HH}$, et al. Predictors of European League Against Rheumatism (EULAR) good response, DAS-28 remission and sustained responses to TNF-inhibitors in rheumatoid arthritis: a prospective study in refractory disease. Springerplus 2015;4:207.

41. Turina MC, Ramiro S, Baeten DL, et al. A psychometric analysis of outcome measures in peripheral spondyloarthritis. Ann Rheum Dis 2016;75:1302-7.

42. Coates LC, Fransen J, Helliwell PS. Defining minimal disease activity in psoriatic arthritis: a proposed objective target for treatment. Ann Rheum Dis 2010;69:48-53.

43. Coates LC, Abraham S, Tillett W, et al. Thu0403 performance of modified minimal disease activity (mda) criteria in patients with peripheral spondyloarthritis: post-hoc analysis of ability-2. Ann Rheum Dis 2016;75(Suppl 2):334.1-334.

44. Svensson B, Andersson ML, Bala SV, et al. Long-term sustained remission in a cohort study of patients with rheumatoid arthritis: choice of remission criteria. BMJ Open 2013;3:e003554.

45. Update on EULAR recommendations for the treatment of RA with DMARDs. Annual European Congress of Rheumatology. London, UK, 2016.

46. Aletaha D, Funovits J, Breedveld FC, et al. Rheumatoid arthritis joint progression in sustained remission is determined by disease activity levels preceding the period of radiographic assessment. Arthritis Rheum 2009;60:1242-9.

47. Lillegraven S, Prince FH, Shadick NA, et al. Remission and radiographic outcome in rheumatoid arthritis: application of the 2011 ACR/EULAR remission criteria in an observational cohort. Ann Rheum Dis 2012;71:681-6.

48. Emery P, Burmester GR, Bykerk VP, et al. Evaluating drug-free remission with abatacept in early rheumatoid arthritis: results from the phase 3b, multicentre, randomised, active-controlled AVERT study of 24 months, with a 12-month, double-blind treatment period. Ann Rheum Dis 2015;74:19-26.

49. van der Woude D, Visser K, Klarenbeek NB, et al. Sustained drugfree remission in rheumatoid arthritis after DAS-driven or non-DASdriven therapy: a comparison of two cohort studies. Rheumatology 2012;51:1120-8.

50. Song IH, Haibel H, Poddubnyy D, et al. Withdrawal of biologic therapy in axial spondyloarthritis: the experience in early disease. Clin Exp Rheumatol 2013;31:S37-42.

51. Burmester GR, Matucci-Cerinic M, Mariette X, et al. Safety and effectiveness of adalimumab in patients with rheumatoid arthritis over 5 years of therapy in a phase $3 \mathrm{~b}$ and subsequent postmarketing observational study. Arthritis Res Ther 2014;16:R24.

52. Burmester GR, Landewé R, Genovese MC, et al. Adalimumab long-term safety: infections, vaccination response and pregnancy outcomes in patients with rheumatoid arthritis. Ann Rheum Dis 2017;76:414-7

53. Burmester GR, Panaccione R, Gordon KB, et al. Adalimumab: longterm safety in 23458 patients from global clinical trials in rheumatoid arthritis, juvenile idiopathic arthritis, ankylosing spondylitis, psoriatic arthritis, psoriasis and Crohn's disease. Ann Rheum Dis 2013;72:517-24. 